\title{
ON THE AMPLITUDE INTERFEROMETER PROGRAM AT THE UNIVERSITY OF MARYLAND*
}

Douglas G. Currie

Department of Physics and Astronomy, University of Maryland, College Park, Maryland, 20742, U.S.A.

\section{ABSTRACT}

A coordinated program of instrumental development and astronomical observation in Amplitude Interferometry has been conducted at the University of Maryland for the past ten years. The objective of this overall program will be presented. The method of operation and the fringe detection technique of the University of Maryland Amplitude Interferometer will be described and reviewed from both the theoretical and the instrumental point of view. The relationship between the detection technique of Amplitude Interferometry and the various parameters of the atmospheric model will be described and the measured Modulation Transfer Function of the telescope/atmosphere will be illustrated and compared with the MTF obtained in other techniques. Samples of the data obtained during the Amplitude Interferometry observing program of the past six years will be presented. This has operated to sixth magnitude, and has reproducibility resolved objects to 0.011 arc seconds with precision of better than 0.002 arc seconds. The reproducibility and stability of repeated measurements of the stellar diameters will be presented. The various uses of this data in astronomy will be briefly described.

The future application of Amplitude Interferometry will be described, particularly a system to obtain high sensitivity and very high resolution with the same detection system and same signal processing as the current instrument. However, for the immediate future, the two instrumental questions are being addressed separately in the form of the Multi-Aperture Amplitude Interferometer, which operates on a conventional telescope and has an increased data rate (by a factor of 8,000 ) and the Very Long Baseline Amplitude Interferometer with

* The manuscript of this paper was not received in time for inclusion in the Proceedings. 
the increased resolution. These instruments and their current status will be described as well as their relationship to the earlier program and their eventual use in a Multi-Aperture Very Long Baseline Amplitude Interferometer system. Finally the possible applications of these techniques of Amplitude Interferometry to a satellite system will be briefly mentioned. 